\title{
Laser Assisted Au Nanocrystal Formation in Conditions of Surface Plasmon Resonance
}

\author{
L. Fedorenko*, B. Snopok, M. Yusupov, O. Lytvyn and Yu. Burlachenko \\ V. Lashkaryov Institute of Semiconductor Physics, NAS of Ukraine, Kyiv, Ukraine
}

The possibility of the calibrated $\mathrm{Au}$ nanoparticles formation in conditions of surface plasmon resonance at irradiation of the $\mathrm{Au}$ film/glass structure by pulsed YAG: $\mathrm{Nd}^{3+}$-laser radiation is investigated. Sizes of the $\mathrm{Au}$ fragments and their surface distribution were studied by the atom force microscopy. The effect of $\mathrm{Au}$ nanofragmentation was observed only at the second harmonic irradiation $(\lambda=0.532 \mu \mathrm{m})$. The large difference in the average values of Au nanoparticles sizes $\bar{\delta}$ and distributions between created in the resonance conditions, $R=R_{\min }, \bar{\delta} \sim 80 \mathrm{~nm}$ and in case out of resonance $R \neq R_{\min }, \bar{\delta} \sim 1350 \mathrm{~nm}$ was established. A high degree of the height sizes homogeneity of $\mathrm{Au}$ nanoparticles has been shown by the histogram obtained from the atom force microscopy data. There was verified the conclusion about determining contribution of the plasmon subsystem field in nanofragmentation process of the Au film in the SPR conditions at high laser levels.

PACS numbers: 81.05.Bx, 81.16.Mk, 81.07.-b

\section{Introduction}

The laser methods of formation of the metal nanoparticles with control of dimensions of Au nanoparticles have been developed on the base of a plasmon resonance effect with size reduction [1] and increasing [2]. At the same time, the surface plasmon resonance (SPR) effect has not been used until in laser technology, but widely used in researches of molecular monolayers in biophysics and biomedicine. The testing of layers is performed by a position of a reflected laser beam intensity minimum $R_{\text {min }}$ in angular dependence $R(\theta)$ for a system molecular layer/film/glass in Kretschmann configuration [3]. Usually, the power of the laser beam in such measurements does not exceed some $\mathrm{mW}$. With increase of laser power the metal film fragmentation will be possible according to the plasmon-polariton wave (PPW) regularities. The aim of this work was to study the possibility of nanofragmentation of the metallic film due to self-organizing micro-ablation process stimulated by high energy plasmon wave excited by YAG-laser in SPR conditions in Kretschmann configuration.

\section{Surface plasmons excitation in the metal film/glass structure at high laser levels}

As it is known [4], excitation of the PPW on the metal film surface is possible under condition of negative real part of the metal dielectric function

$$
\varepsilon_{m}^{\prime}=\varepsilon_{\infty}-\omega / \omega_{\mathrm{sp}}^{2},
$$

where $\omega_{\mathrm{sp}}=\omega_{\mathrm{p}} /\left(1-\varepsilon_{1}\right)^{1 / 2}$ - surface plasmon frequency,

\footnotetext{
* corresponding author; e-mail: lfedor@isp.kiev.ua
}

$\omega_{\mathrm{p}}=\left(4 \pi n e^{2} / m\right)^{1 / 2}$ - volume plasmon frequency, $\varepsilon_{1}$ dielectric function of air. Equation (1) will be carried out in case $\omega<\omega_{\mathrm{sp}}$, inset, Fig. 1. Maximum of laser energy is transformed into plasmon subsystem in the conditions of SPR when intensity of reflected beam tends to zero, Fig. 1. Au film fragmentation is possible if the displacement $l$ of PPW image along a surface during $t_{\mathrm{p}}$ is much less than plasmon wavelength $\lambda_{\mathrm{sp}}, l \ll \lambda_{\mathrm{sp}}$. As one can see from inset in Fig. 1, such condition can be realized when the PPW group velocity $V_{\mathrm{g} x}$ :

$$
V_{\mathrm{g} x}=\partial \omega / \partial k_{x} \rightarrow 0 \text {. }
$$

This can be achieved at large values of a wave vector $k_{x}=(\omega / c)\left[\varepsilon_{1} \varepsilon_{2} /\left(\varepsilon_{1}+\varepsilon_{2}\right)\right]^{1 / 2}$, where $\varepsilon_{1}$ - dielectric function of the air, $\varepsilon_{2}-$ dielectric function of metal. It is possible at $\left|\varepsilon_{1}\right| \rightarrow \varepsilon_{2}$, i.e. in a right part of the $\omega(k)$ dependence in Fig. 1.

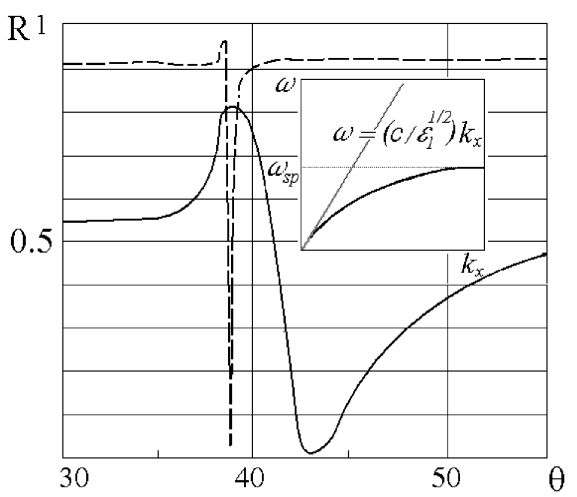

Fig. 1. Dependence $R(\theta)$ for $\mathrm{Au} /$ glass structure calculated according to [5]. Dashed $\lambda=1.064 \mu \mathrm{m}$, solid $\lambda=0.532 \mu \mathrm{m}$, Au film thickness $h=40 \mathrm{~nm}$ PPW excitation on the metal film surface in Me/glass system. 


\section{Experimental configuration}

System Au film/glass wafer was irradiated by the fundamental $\left(\lambda=1.064 \mu \mathrm{m}, t_{\mathrm{p}}=10 \mathrm{~ns}\right)$ and the second $(\lambda=0.532 \mu \mathrm{m})$ harmonics of the Q-switch YAG: $\mathrm{Nd}^{3+}$ laser 1 , polarized in a plane of incidence $(\mathrm{p}$ - polarization) in Kretschmann configuration, Fig. 2. The optical scheme of SPR included: semi-cylindrical prism 2, photodiode 3 , immersion layer 4 , glass wafer 5 with $\mathrm{Au}$ thin film $6, \delta \sim 40 \mathrm{~nm}$, deposited by a standard method. The glass wafer 7 was installed through an air gap from a gold film, and was used as a collector of Au particles reaching it due to microablation process. The control of reflected laser beam intensity was carried out by the photodiode 3. Experimental setup contained the rotating mechanism and scanning $2 \mathrm{D}$ coordinate $X-Z$ system to measure the angular dependence $R(\theta)$ and also for uniform laser processing of $\mathrm{Au} /$ glass boundary in a scanning mode. The system Au film/glass was irradiated from a side of glass substrate through a prism and immersion layer, Fig. 2. Atom force microscopy (AFM) images and optical absorption spectra were used to research the surface morphology and for an additional control of plasmon resonance frequency. The calculated values of incidence angle $\theta$ for SPR case, according to [5] and determined experimentally are well coincided.

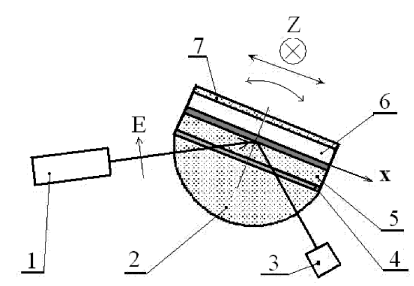

Fig. 2. Layout of SPR experimental setup.

\section{Results and discussion}

AFM images of the $\mathrm{Au}$ film surface at irradiation by pulses of the second harmonic YAG: $\mathrm{Nd}^{3+}$ laser $(\lambda=$ $\left.0.532 \mu \mathrm{m}, t_{\mathrm{p}}=10 \mathrm{~ns}\right)$ at different angles of incidence are shown in Fig. 3. It is obvious that in the case (a) (out of SPR conditions) Au particles had the average size $\bar{\delta} \sim 1350 \mathrm{~nm}$ with surface density $n_{\mathrm{s}} \sim 3 \times 10^{8} \mathrm{~cm}^{-2}$. In case (b) (in SPR) the average size of particles $\bar{\delta}$ was essentially smaller, namely $\bar{\delta} \sim 80 \mathrm{~nm}$ with near circular form, the surface density was $n_{\mathrm{s}} \sim 5 \times 10^{9} \mathrm{~cm}^{-2}$ and they had rather uniform distribution on the surface. Energy density threshold $P_{\text {thn }}$, at which $\mathrm{Au}$ film structurization starts in SPR conditions, was appreciably lower $P_{\text {thn }} \approx 5.5 \mathrm{~J} \mathrm{~cm}^{-2}$ than the $P_{\text {thn }}$ in the case out of SPR conditions, $P_{\text {thn }} \approx 8 \mathrm{~J} \mathrm{~cm}^{-2}$. The high degree of nanofragments height homogeneity is demonstrated by the histogram in Fig. 4 obtained from AFM image data, Fig. 3b. The spectral transmission data, Fig. 5, confirm the reasonability of the second harmonic YAG: $\mathrm{Nd}^{3+}$ laser choice so as the frequency is close to resonance $\omega_{\mathrm{sp}}$ for surface PPW. Besides, spectra mode indicates that in the case of the nanocrystallites formation in SPR conditions $\mathrm{PPW}$ is radiative close to the transmission maximum.

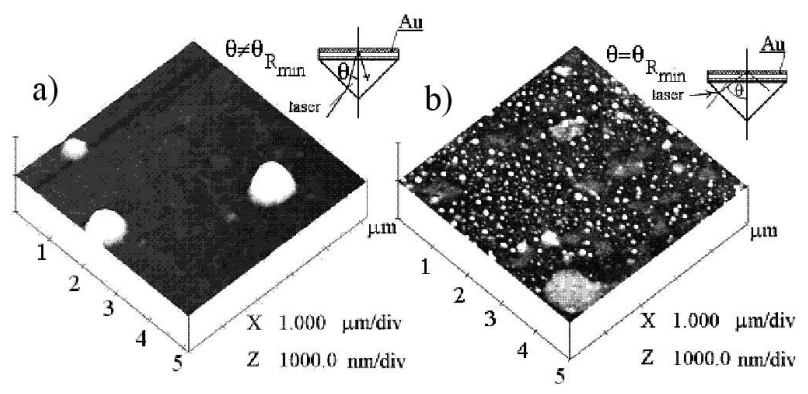

Fig. 3. AFM images of the Au film surface at irradiation by pulses of the second harmonic YAG: $\mathrm{Nd}^{3+}$ laser $\left(\lambda=0.532 \mu \mathrm{m}, t_{\mathrm{p}}=10 \mathrm{~ns}\right)$ at the angle of incidence $\theta$, (a) $\angle \theta \sim 10^{\circ}$ (out of SPR) and (b) $\angle \theta=41.3^{\circ}$ ( $R=R_{\min }$, in $\mathrm{SPR}$ conditions).

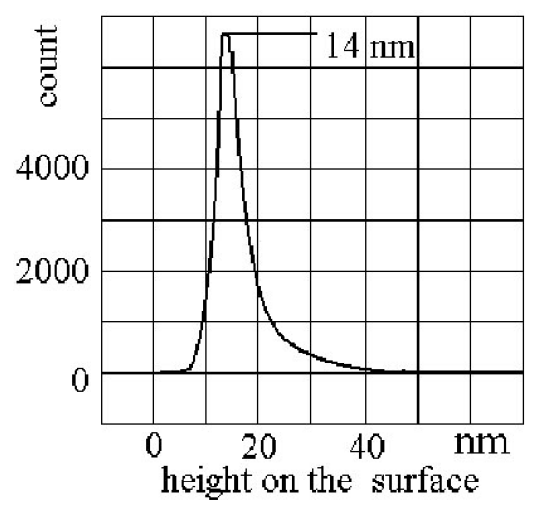

Fig. 4. Histogram of the Au nanofragments height calculated from AFM image (Fig. 3b).

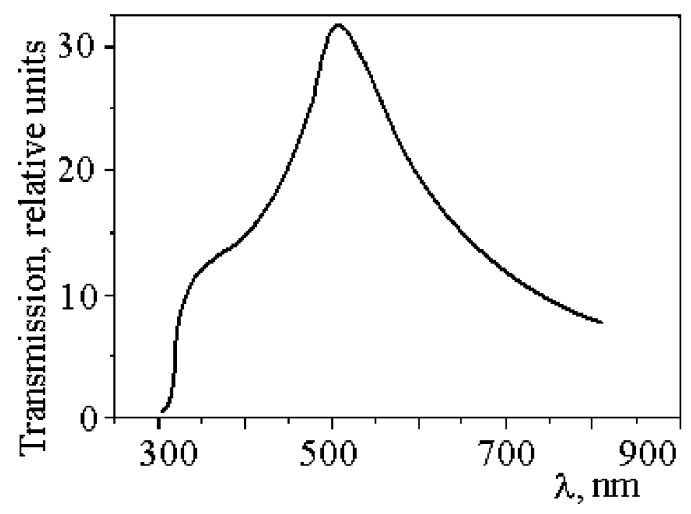

Fig. 5. Transmission spectrum of the structure $\mathrm{Au} /$ glass, appropriate Fig. 3b.

Nanofragmentation of the Au film on glass substrate in the SPR conditions can be explained by action of 
the plasmon subsystem on the phase transition processes (melting and consequent micro-ablation). At laser frequencies close to $\omega_{\mathrm{s}}$ the contribution of plasmon component in the PPW increases. In this case the PPW has the group velocity $V_{\mathrm{gr}} \rightarrow 0$, therefore the PPW field image is practically fixed on the surface during $t_{\mathrm{p}}$. The PPW is converted to an ensemble of local excitations, with energy distribution according to the plasmon field along the surface [4]. With increasing laser intensity in resonance conditions, plasmon excitations accumulate a large energy enough to phase transition with a period $x_{\mathrm{pl}} \sim n \lambda_{\mathrm{s}} / 2$ along a surface, where $n$ is integer. That, apparently, ensures fragmentation of $\mathrm{Au}$ film with discreteness appropriate to a characteristic distance of the plasmon subsystem. Mentioned above, were confirmed by the experimental fact of the absence of similar nanofragmentation effect under irradiation by the fundamental harmonic of YAG: $\mathrm{Nd}^{3+}$ laser $(\lambda=1.064 \mu \mathrm{m})$ in the SPR conditions. In this case the condition (2) is defaulted, as the excitation frequency corresponds to a central part of $\omega(k)$ dependence (inset, Fig. 1).

\section{Conclusions}

The advantage of the presented method consists in a capability of calibration of the nanoparticles sizes depending on laser wavelength. This is possible up to limit defined by degree of the PPW spreading. The observed effect has a perspective of use for generation of nanosize particles both on an initial surface, and on some deleted from it and specially prepared, similar to the laser induced forward transfer (LIFT) method [6].

\section{Acknowledgments}

The authors are grateful to Prof. M.L. Dmitruk and Prof. L.I. Berejinskyi for the useful discussions during the fulfillment of activity.

\section{References}

[1] A. Takami, H. Kurita, S. Koda, J. Phys. Chem. B 103, 1226 (1999).

[2] N. Chandrakharan, P.V. Kamat, J. Hu, G. Jones, J. Phys. Chem. B 104, 11103 (2000).

[3] L.M. Nosach, P.M. Boltovets', O.Iu. Povnytsia, V.L. Zhovnovata, O.M. Zakharenko, B.A. Snopok, Iu.M. Shyrshov, N.S. Diachenko, Mikrobiol. Z. 67, 58 (2005).

[4] Surface Polaritons, Eds. V.M. Agronovich, D.L. Mills, North Holland, Amsterdam 1982, p. 585.

[5] R.M.A. Azzam, N.M. Bashara, Ellipsometry and Polarized Light, Mir, Moscow 1981, p. 387.

[6] W.L. Barnes, T.W. Preist, S.C. Kitson, J.R. Sambles, Phys. Rev. B 54, 6227 (1996).

[7] V.P. Veiko, A.I. Kaidanov, H.A. Kovachki, E.A. Shakhno, Conference Proceedings Paper, Laser Radiation Photophysics, Bodil Braren; Mikhail N. Libenson, Editors, 1856, 11 (1993). 